\title{
New WHO medical eligibility criteria for contraceptives: adaptation for use in a local service in UK
}

\author{
Paul O'Brien, MSc, MFFP, SCMO Service for Women
}

\author{
Correspondence: Dr Paul O'Brien, Parkside Heath, Raymede Clinic, Exmoor St, London, W10 6DZ, UK \\ paulobrien@care.prestel.co.uk
}

The Journal of Family Planning and Reproductive Health Care 2001: 27(3): 149-152

\section{Introduction}

The World Health Organization (WHO) has published a new edition of its influential document Improving Access to Quality Care in Family Planning: Medical Eligibility Criteria for Contraceptives. The first edition, published in 1996, has had a large impact and has been used in many countries as a framework for the production of national and service guidelines. The aim of the WHO initiative is to establish international norms for the medical eligibility criteria for contraceptive methods. The new edition updates advice where new evidence has emerged. ${ }^{1}$

The medical eligibility criteria are based on the work of a group of international experts who reviewed all the clinical evidence on methods of contraception-looking for the circumstances in which it is safe or unsafe to use a method. They developed a new system for classifying the medical eligibility criteria based on balancing the potential benefits and harms.

Part of the popularity of this resource is that it presents the information in a user-friendly format, helping clinicians to provide rational advice to clients choosing the most suitable method for them. We have adapted it for use in a community contraceptive service in London.

\section{Methods used by WHO}

For the first edition a number of agencies and organisations collaborated in an in-depth review of the epidemiological and clinical evidence relevant to medical criteria of eligibility for well-established contraceptive methods. The process involved comparing the eligibility criteria used by different agencies for various contraceptives, preparing summaries of the relevant published medical and epidemiological literature, and preparing a draft classification for review by a larger group of experts and agencies.

At subsequent meetings a new approach to categorise the suitability of different contraceptive methods in the presence of specific illnesses or conditions was developed. The categorisation was achieved by weighing the health risks and benefits of using a particular contraceptive method when any of those conditions is present. The evidence was reviewed again last year, and new data were taken into account in the latest edition. The published summary of conclusions and recommendations is a consensus achieved amongst the experts and agencies.

The medical eligibility criteria are designed to protect women from the potential adverse effects of contraceptives, while ensuring that they are not denied a choice of suitable methods, with an adequate margin of safety. The classification scheme chosen to present the criteria is simple and useful, and avoids the traditional terms like 'contraindications', 'cautions' and 'warnings', which we see in the

British National Formulary (BNF) and Summary of Product Characteristics (SPC) in the UK, and which are open to different interpretations.

Unusually, WHO provides a list of conditions in which there should be no restriction in the use of a method. This is useful for clinicians when faced with a condition or disease with which they are unfamiliar, or are unsure about its appropriateness. If we move to nurse prescribing of hormonal contraceptives, WHO eligibility criteria will be an invaluable resource.

\section{Classification scheme}

Each condition was defined as representing either an individual's characteristics (for example, age or pregnancy history), or a known pre-existing medical or pathological condition (for example, diabetes or hypertension). The clinical history, and sometimes examination, are used to screen for the characteristic or condition. The conditions affecting eligibility for the use of each contraceptive method were classified under one of the four categories in Table 1.

Classes 1 and 4 are self-explanatory. What might in the past have been called 'relative contraindications' are developed more fully, and are divided into 'generally used' and 'not usually recommended' categories. Classification of a method/condition as category 2 indicates the method can generally be used, but careful follow-up may be required. However, provision of a method to a woman with a condition classified as category 3 requires careful clinical judgement and access to clinical services; for such a woman, the severity of the condition and the availability, practicality, and acceptability of alternative methods should

Table 1 WHO Classification scheme

\begin{tabular}{ll}
\hline Classification category & Clinical interpretation \\
\hline $\begin{array}{l}\text { 1. A condition for which there is no } \\
\text { restriction for the use of the } \\
\text { contraceptive method. }\end{array}$ & $\begin{array}{l}\text { Use method in any } \\
\text { circumstances }\end{array}$ \\
$\begin{array}{l}\text { 2. A condition where the advantages } \\
\text { of using the method generally } \\
\text { outweigh the theoretical or proven } \\
\text { risks. }\end{array}$ & Generally use the method \\
$\begin{array}{l}\text { 3. A condition where the theoretical or } \\
\text { proven risks usually outweigh the } \\
\text { advantages of using the method. }\end{array}$ & $\begin{array}{l}\text { Use of method not usually } \\
\text { recommended unless other } \\
\text { more appropriate methods are } \\
\text { not available or not }\end{array}$ \\
$\begin{array}{l}\text { 4. A condition which represents an } \\
\text { unacceptable health risk if the } \\
\text { contraceptive method is used. }\end{array}$ & \begin{tabular}{l} 
Method not to be used \\
\hline
\end{tabular}
\end{tabular}


Table 2 Examples of conditions, classification and comments from $\mathrm{WHO}$

Condition Class Comments by WHO

\section{COC}

Past ectopic pregnancy

Smoking

a) Age $<35$ years

b) Age $\geq 35$ years

(i) $<15$ cigarettes/day

(ii) $\geq 15$ cigarettes/day

Obesity

$\geq 30 \mathrm{~kg} / \mathrm{m}^{2}$ body mass index (BMI)

Obesity is a risk factor for venous thromboembolism.

\section{IUD and LNG-IUD}

Uterine fibroids

a) Without distortion of the uterine cavity

b) With distortion of the uterine cavity against ectopic pregnancy. increasing number of cigarettes smoked per day.

The risk of future ectopic pregnancy is increased among women who have had an ectopic pregnancy in the past. COCs provide protection

Risk of cardiovascular events increases with increasing age and

be taken into account. For a method/condition classified as category 3 , use of that method is not usually recommended unless other more appropriate methods are not available or acceptable. It should be the method of last choice. Careful follow-up will be required.

The guidance is presented in a series of tables, one for each method type, with a list of conditions and diseases, the advice given which is based on a balance of benefit and risk, and the reason for the advice. Some examples of the classification scheme and comments from WHO are given in Table 2.

The category assigned to a condition is based on estimation of the chance of exacerbating a theoretical or demonstrated risk associated with a specific illness or condition, which is balanced against the contraceptive benefit. For clients with multiple risks, the category assigned to the risk conditions under each method may be changed to reflect the need for more caution, although more than one risk will not necessarily change the category.

\section{Initiation and continuing use}

On occasion, WHO recommendations for some conditions distinguish eligibility criteria for initiating and continuing use of the method. They may differ because minor method side effects can mimic serious health problems. For example, for copper IUDs and LNG-IUD, unexplained vaginal bleeding is a Class 4 condition for initiating use, but a Class 2 condition for continuing use since bleeding pattern changes are a common side effect of the methods. Alternatively, certain serious health problems could be method-related. Migraine with focal neurological symptoms are a Class 2 condition for women initiating use of progestogen-only methods, but a Class 3 condition when it develops in current users, primarily because of concern that use of progestogen might have contributed to the condition.

\section{Changes in the new edition}

Some changes have been made for the new edition. The most important are the ones which have changed from Class 1 or 2 (generally safe to use) to Class 3 or 4 (generally not safe to use), or vice versa. Mild hypertension is now Class 3 for use of the combined pill where it was Class 2. Indeed, for women with moderate hypertension the experts have taken a more cautious approach and have moved all hormonal methods up a class. Of the progestogen methods only in the case of injectables has this change been from Class 2 to Class 3 . The upgrading of hypertension as a risk factor for hormonal methods follows a number of recent epidemiological studies showing the significance of a history of elevated blood pressure as a risk factor for cardiovascular morbidity.

For the initiation of oral methods, unexplained vaginal bleeding was Class 3 and in the latest edition is Class 2. Fibroids that distort the uterine cavity were Class 2 for intrauterine devices or the LNG-IUD use, but are now given a Class 4, as proper IUD placement might not be possible.

UK readers will be familiar with this scheme of classification which was described in the guidelines from the Faculty of Family Planning and Reproductive Health Care on First prescription of combined oral contraception: recommendations for clinical practice. ${ }^{2}$ The latest edition from WHO introduces a few changes from those in the Faculty guidance.

\section{Adaptation for use in a local community contraceptive service}

The WHO document aims to provide guidance to family planning and reproductive health programmes, and the scientific community, in the preparation of guidelines for service delivery of contraceptives. It does not provide rigid guidelines, but rather gives recommendations for rationalising the provision of various contraceptives in view of the most up-to-date information available on the safety of the methods, as understood by a group of international experts. The authors stress that the document should not be seen or used as the actual guidelines, but rather as a reference.

We have made a number of changes to suit the circumstances of our service in West London. One obvious one is in the COC guidance removing any reference to circumstances when blood pressure measurement is not available. This was developed for use in community family planning programmes where full clinical assessment is not available. It could also be used in some European countries 
where COCs are available over the counter at pharmacies. The vast majority of recommendations agree with typical UK practice and can be adopted directly. These are examples of the few areas of COC prescribing where there may be differences.

\section{Breast feeding}

Many practitioners in the UK do not prescribe the combined pill as long as a woman is breast-feeding. The SPC for Microgynon-30 states that 'Mothers who are breast-feeding may be advised instead to use a progestogen-only pill.' WHO classify COC use from 6 weeks to 6 months postpartum as Class 3, and after the first 6 months as Class 2. The experts do not indicate why Class 2 rather than Class 1 after 6 months - because of lesser effects of the COC on the duration and/or quality of the milk at this age, or because most infants are less reliant on breast milk.

\section{Age}

Since the publication of epidemiological data showing that any excess risk of arterial diseases in combined pill users is largely confined to smokers, we have been more inclined to continue use of the pill beyond 35 years. However, the risk of cardiovascular events increases with age and this is reflected in the advice in the BNF which suggests using the COC with caution over 35 years and avoiding in women over 50. WHO has suggested a later threshold, and recommends no restriction in use of the combined pill up to 39 years of age (Class 1), has given 40 year and above Class 2 in the absence of other adverse clinical conditions, with the same class continuing until the menopause. This suggests that the discussion of the age-related increase in cardiovascular risk could be initiated around 40, with no upper limit in its use if the woman understands the small risks and does not have other cardiovascular risk factors.

\section{Smoking}

The UK SPC for Microgynon discusses risk without giving prescribing advice: 'The risk of venous and/or arterial thrombosis associated with combined oral contraceptives increases with smoking; with heavier smoking and increasing age the risk further increases, especially in women over 35 years of age.' The BNF advises that women who smoke should use the combined pill with 'caution' and 'avoid' it if smoking 40 or more cigarettes a day. The WHO advise that for women 35 years and over, the use of less than 15 cigarettes per day is Class 3 , and 15 or more cigarettes Class 4 . This gives some latitude to the prescriber when a light or moderate smoker finds other methods unsuitable, or where a woman declines other methods.

\section{Family history of venous thromboembolism}

A family history of deep venous thrombosis or pulmonary embolism (defined by WHO as occurrence in a first-degree relative), is given as Class 2 for the use of the combined pill. There has been much discussion on the screening of women with family histories of venous thrombosis, most recently in the review article by Vandenbroucke et al. ${ }^{3}$ They point out that 'even in the absence of the defects [in thrombophilia] already known to be relevant, a strong family history of venous thrombosis warrants caution about the use of oral contraceptives, purely on clinical grounds.' WHO does not address the issue of screening for thrombophilias. The British Society for Haematology has a task force looking at the issue of screening for familial thrombophilias, and we await the new guidelines, which should be published in the autumn.
Trophoblastic disease

The effect of exogenous hormones in patients with persistent trophoblastic activity following a hydatidiform mole is an area of considerable controversy. The WHO expert group advises that there should be no restriction in use of the combined pill in women with a history of benign or malignant gestational trophoblastic disease (Class 1). The advice from the Royal College of Obstetricians and Gynaecologists, on the other hand, is that the pill can be taken safely once the hCG levels, monitored at a reference centre, have returned to normal. ${ }^{4}$ The reason for this discrepancy is that the indications for chemotherapy in the UK are more conservative where oncologists are prepared to follow patients with elevated hCG for between 4 and 6 months before starting chemotherapy. In the United States, and possibly many other countries, the policy is to treat patients at 8 weeks if their hCG is still elevated.

Epidemiological evidence from the US suggests that starting the pill early does not increase the incidence of women requiring chemotherapy. Data from the UK, on the other hand, suggests that there may be a subgroup of women with more prolonged and persistent trophoblastic activity where disease can be stimulated by the early starting of hormonal contraceptive products. ${ }^{5}$ This group would be monitored in the UK, but treated with chemotherapy in the US. The result is that the proportion of women receiving chemotherapy in the US is around $30 \%$, but around $10 \%$ in the UK.

The WHO advice reflects the North American experience and in our service we will continue to follow the RCOG advice and not recommend initiating COC use until hCG levels have returned to normal.

\section{Drug interactions}

There is considerable variation in opinion about the importance of any interaction between COCs and broadspectrum antibiotics. ${ }^{6}$ WHO makes no mention of reduced efficiency in women using non-enzyme-inducing antibiotics. In discussing drug interaction the BNF says that some broadspectrum antibiotics (e.g. ampicillin, doxycycline) may reduce the efficacy of combined oral contraceptives, but states that the risk is probably small. The UK fpa's advice is that additional contraceptive precautions should be used whilst taking a short course of a broad-spectrum antibiotic and for 7 days after stopping. For practical reasons our service will continue to follow the fpa's advice.

\section{Prescriber accountability}

As with all guidelines, our eligibility criteria are not a blueprint for practice. We intend that they will be used to inform decision making for our clients, knowing we have the recommendations from WHO and the support of our employer. As always, with guidelines of this sort, clinicians are at liberty - after full discussion with the individual woman - to deviate from them, in which case we ask them to record the thinking behind the decision and understand that they are accountable for this, as well as their other prescribing decisions.

\section{Dissemination and implementation}

We have introduced the new medical eligibility criteria to our clinical staff at a clinical meeting, and the new guidelines are being circulated to all clinics for inclusion in our existing Practice Guidelines. Some of the guidelines will be different from current individual practices, and staff members will need support in implementing them. We encourage feedback on their use and will review the guidelines 1 year after introduction. 
WHO guidance and evidence-based medicine

Why have guidelines? One reason often cited is the wide variation in clinical practice. Little is known about the variation in medical eligibility criteria in the UK. A survey of national family planning organisations found that the level of blood pressure that would contraindicate the use of the combined pill varies widely between countries. ${ }^{7}$ Some of these variations will reflect local circumstances and resources. WHO has varied the recommendations when facilities for monitoring blood pressure are limited. Some, however, will reflect the differing interpretations of the research evidence and their translation into practice guidelines. Evidence-based guidelines limit unjustified variations in practice by systematically assessing the research evidence and formulating advice for best practice directly related to the evidence, where it exists, and being explicit about the rationale for recommendations where it does not.

Much work has been done on guidelines development over the past 10 years. Components of an evidence-based guideline development programme include selecting participants, locating, evaluating and grading the evidence, and formulating and reviewing the guidelines. ${ }^{8}$ Identifying best practice is dependent on the whole development process. Although WHO suggests that these recommendations are not actual guidelines but 'guidance' to be adapted locally, the lessons learned about the importance of the process of guideline development should apply to any such authoritative recommendations.

For the first edition of Improving Access the recommendations were based on expert assessment of commissioned narrative reviews which have not been published. Selected references were cited in support of the recommendations, but the relationship between the references and the recommendations was not clear. The Working Group used informal methods to appraise the evidence and generate the recommendations, but these also were not described. For the latest edition new evidence since 1995 has been critically appraised and graded. A systematic review of the evidence will be published in a peer-reviewed journal (Herbert Peterson, WHO, personal communication). Informal consensus methods were used to derive the recommendations, but these have not been described. In Improving Access WHO has published the summary and recommendation of the deliberations of the Working Groups in an easy-to-use tabular format. More attention needs to be paid to the development process used in the production of these recommendations. While a systematic process was used to synthesise the new evidence for the latest edition, the same rigour needs to be applied to the pre1995 evidence supporting the earlier recommendations. The synthesis of non-randomised evidence, the bulk of the safety data in contraception, has not been standardised and conclusions drawn may vary.

The systematic review will tell us the strength of some of the evidence and how it was synthesised. The next stage linking the recommendations to the evidence - also needs thorough reporting to give those adapting and using the recommendations the confidence that the advice reflects best practice. Though interpreting evidence inevitably involves value judgements, by making the process explicit the scientific basis of the judgements should be made as clear as possible. The WHO assignment of Class 4, or for that matter Class 1, does not indicate the strength of the body of supporting evidence or the strength of the recommendation, but provides the results of an assessment by experts of the scale of the risk, balanced against any benefit. The NHS Executive in the UK requires that all guidelines carry a classification which tells whether the guidance is supported by research evidence or is more in the nature of expert opinion. ${ }^{9}$ We should be told which advice from the WHO is supported by research evidence, the strength of the evidence, and which recommendations are based in expert opinion alone. In the absence of strong evidence, expert opinion is appropriate. Users need to know that the make-up of the WHO review Working Group or personalities did not have an undue influence on the deliberations. Formal consensus methods improve the validity of the recommendations where the evidence base is weak. ${ }^{10}$

It is highly unlikely that we will ever be able to evaluate many of the WHO recommendations in terms of improvement in health outcomes. A prospective assessment of the validity (the extent to which they lead to the health outcome projected) of the recommendations can be made by consideration of the substance and quality of the evidence cited, the means used to evaluate the evidence and the relationship between the evidence and the recommendations. ${ }^{11}$ However, to do this the procedures followed in developing the recommendations, the participants involved, the evidence used, the assumptions and rationales accepted, and the analytic methods employed, must be meticulously documented and described. ${ }^{11}$

Despite these criticisms Improving Access to Quality Care in Family Planning is based on consideration of reviews and discussion of the evidence by international experts, with participants from 21 countries, including representatives from several agencies, organisations and women's health advocates. In the absence of explicit evidence-based guidance, the WHO has produced what is generally perceived to be the most authoritative set of recommendations of medical eligibility criteria for contraceptive methods.

\section{Conclusions}

The WHO guidance is the most authoritative advice on medical eligibility criteria available. The data are presented in a user-friendly format for local application. The publication of the evidence supporting the recommendations and formal linking of advice to the evidence is necessary, and would make their adaptation for local use an easier task. We have used the latest edition to revise our contraception policies and prescribing practices which we hope will help improve access to, and the quality of, family planning services for our clients in West London. The next edition, of what is probably the most influential publications from the Reproductive Health and Research Department of WHO, will be more convincing if it follows a formal guidelines development process.

The full document is available at <http://www. who.int/reproductive-health/ pages_resources/listing family_planning.htm $>$ and single hard copies can be requested from World Health Organization, Department of Reproductive Health and Research, Documentation Centre, 1211 Geneva 27. Orders are also possible by email at $<$ rhrpublications@who.int>.

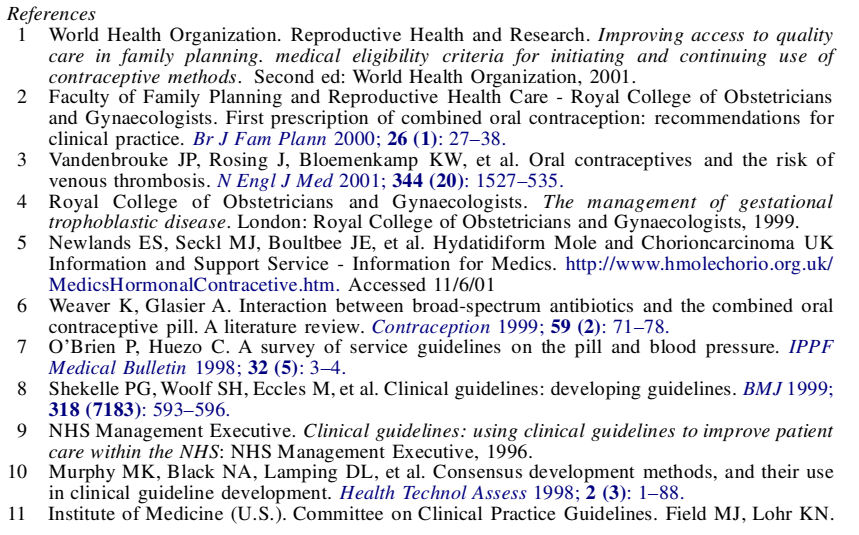

